\title{
DUST DETECTION OVER BRIGHT SURFACES USING HIGH-RESOLUTION VISIBLE SEVIRI IMAGES
}

\author{
Yehia Eissa, Hosni Ghedira, Taha B.M.J. Ouarda and Matteo Chiesa \\ UAE Research Center for Renewable Energy Mapping and Assessment \\ Masdar Institute, Masdar City, PO Box 54224, Abu Dhabi, UAE
}

\begin{abstract}
Reflectance observations from the high-resolution visible (HRV) channel of the SEVIRI instrument, onboard Meteosat Second Generation, have shown to be sensitive to dust over bright surfaces, i.e. bright desert sand. This was observed when deriving the cloud index from the HRV channel for dusty days over the desert environment of the United Arab Emirates (UAE). Sensitivity of the HRV channel to dust could then be used to derive an index giving an indication of the dust conditions in the atmosphere. Therefore, the purpose of this paper is to propose an index, the interception index, for dust detection over bright desert surfaces in the UAE using the HRV SEVIRI channel. The ability of the interception index to estimate the direct normal irradiance is also investigated.
\end{abstract}

Index Terms - Interception index, cloud index, dust detection, HRV channel, direct normal irradiance

\section{INTRODUCTION}

Dust detection over bright surfaces, like bright desert sand, may seem difficult using satellite channels in the visible range of the electromagnetic wave spectrum. That is due to the color similarity between airborne dust particles and the bright sand background. However, when dealing with cloud index $n$, computed using the high-resolution visible (HRV) channel of the SEVIRI instrument, onboard Meteosat Second Generation, it was observed that this visible channel is also sensitive to dust.

The cloud index $n$ measures the thickness of clouds over a pixel having a value ranging from 0 to 1,0 being for clearest sky conditions and 1 for overcast conditions. It has a temporal resolution of 15-min and a spatial resolution of 1$\mathrm{km}$ at nadir, giving it an advantage over other cloud optical depth products derived from polar orbiting satellites. Cloud index $n$ was introduced in the Heliosat model to estimate the surface global horizontal irradiance $(G H I)$ after computing the clear-sky $G H I$ [1-3]. In this model clear-sky $G H I$ already accounts for the turbidity of the cloud-free atmosphere. Therefore, the dust effect on the cloud index $n$ is not favorable in such a case.
Alternatively, cloud index $n$ could be informative of the dust cover over bright surfaces if the cloudy pixels are removed by means of a cloud mask [4]. In this case it would be more appropriate to call it the "interception index".

The objective of this paper is to propose a derivation of the interception index $i$ from the HRV SEVIRI channel and to assess its capability for dust detection over bright desert surfaces such as those in the United Arab Emirates (UAE). The ability of the interception index $i i$ to estimate the direct normal irradiance $(D N I)$ is also investigated.

\section{BACKGROUND ON CLOUD INDEX}

Earlier versions of Heliosat method derived the cloud index $n$ using the digital count measured by the satellite. Currently modified, Heliosat-2, derives the cloud index $n$ using the radiance measured by the SEVIRI instrument, and from that the cloud transmission is computed and is used to estimate the $G H I[2,3]$. Visible channels of geostationary satellites were used in several studies to derive the cloud index $n$ [57].

In this study the cloud index $n$ is derived from the HRV channel of the SEVIRI instrument. As mentioned earlier, the cloud index $n$ is a measure of the thickness of cloud cover over a specific pixel, and is computed by:

$$
n=\frac{\rho^{t}(i, j)-\rho_{\min }^{t}(i, j)}{\rho_{\max }-\rho_{\min }^{t}(i, j)},
$$

where $\rho^{t}(i, j)$ is the instantaneous reflectance over pixel $(i, j)$, $\rho_{\text {min }}^{t}(i, j)$ is the surface albedo and $\rho_{\max }$ is the maximum reflectivity which occurs in highly overcast conditions.

Rigollier et al. [3] suggest that $\rho_{\max }$ depends on the solar zenith angle, while Perez et al. [7] suggest it remains constant throughout as long as the calibration drift in the sensor is accounted for. In this study $\rho_{\max }$ was set as a constant.

In this study $\rho_{\text {min }}^{t}(i, j)$ was computed on a monthly basis to take into account the non-Lambertian nature of the Earth's surface $[2,3]$. Thus, $\rho_{\text {min }}^{t}(i, j)$ was taken as the $4^{\text {th }}$ percentile for a specific scene, location and month since it happens that the pixels in the Meteosat image may record very low radiances due to cloud shadow or when the sun is above the horizon [3]. Using this method is also applicable to the 


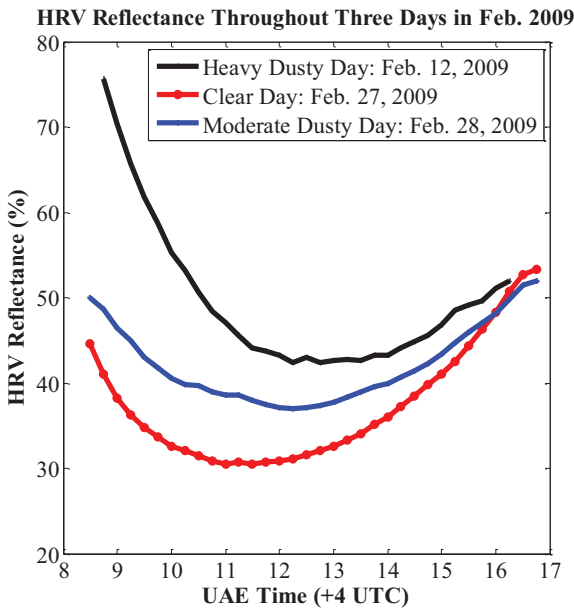

(a)

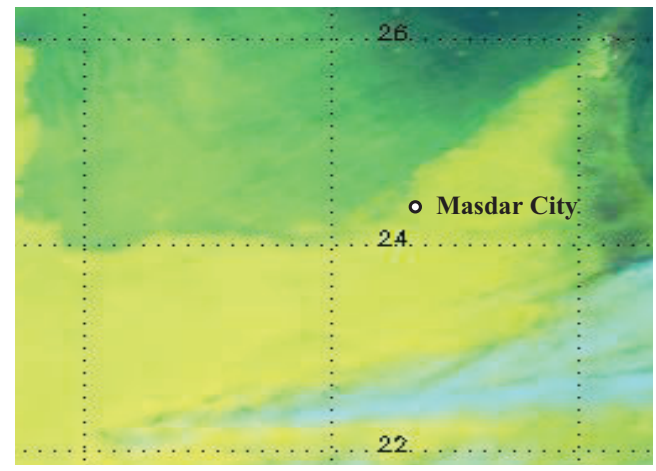

(c)

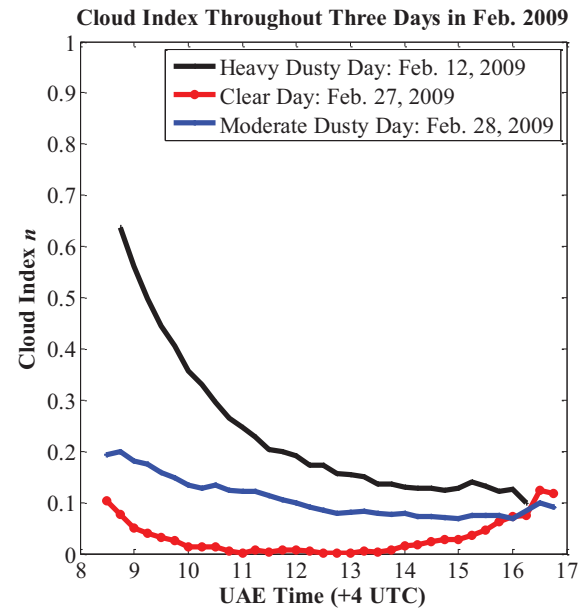

(b)

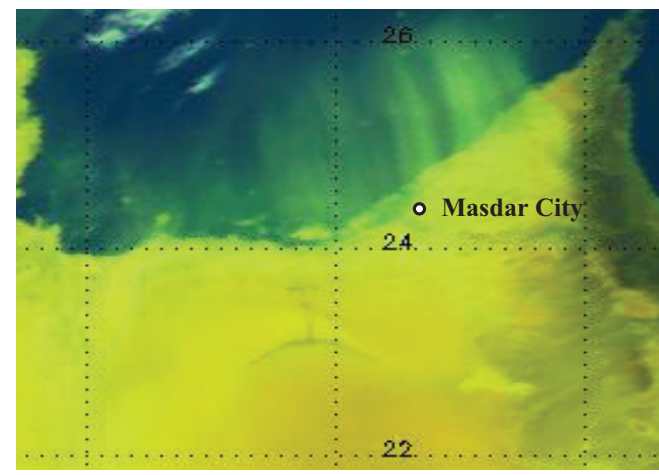

(d)

Fig. 1: The HRV reflectance throughout three days with different weather condition (a); the cloud index throughout the same three days (b); dusty conditions on Feb. 12, 2009 (c); dust conditions on Feb. 28, 2009.

available dataset, since it is unlikely that a pixel is constantly cloudy for a whole month.

The clear-sky index $k_{T}{ }^{*}$ is calculated using the following equations $[2,3]$ :

$n \leq-0.2, \quad k_{T}^{*}=1.2$,

$-0.2<n \leq 0.8, \quad k_{T}^{*}=1-n$,

$0.8<n \leq 1.1$,

$k_{T}^{*}=2.0667-3.6667 n+1.6667 n^{2}$,

$1.1<n, k_{T}^{*}=0.05$.

Modeled $G H I$ reaching the surface is then calculated by:

$G H I=k_{T}^{*} G H I_{\text {clear }}$,

where $G H I_{\text {clear }}$ is the clear-sky, i.e. cloud-free, $G H I$ which takes the atmospheric turbidity into account.

\section{THE INTERCEPTION INDEX}

\subsection{Sensitivity of HRV channel to dust}

Fig. 1a shows the HRV reflectance throughout three days in Feb. 2009 and Fig. 1b shows the derived cloud index $n$ for the same three days. This figure clearly shows the sensitivity of the HRV channel to the dusty conditions present in Feb. 12, 2009 shown in Fig. 1c and Feb. 28, 2009 shown in Fig. $1 \mathrm{~d}$.

\subsection{Proposed method to derive the interception index}

By applying the cloud masks presented in Hocking et al., it was observed that the thin cirrus test applies a cloud mask using the brightness temperature readings of the T09 and T10 channels of SEVIRI, and does not classify dusty pixels as cloudy [4]. The conditions to classify a pixel as cloudy: $\mathrm{T} 09-\mathrm{T} 10>\mathrm{T} 09_{\mathrm{b}}-\mathrm{T} 10_{\mathrm{b}}+\Delta \mathrm{T}$,

T09<303.15K,

where T09 and T10 are the brightness temperature values observed for the T09 and T10 channels, respectively, T09 and $\mathrm{T} 10_{\mathrm{b}}$ correspond to the background clear sky brightness temperatures for the T09 and T10 channels, respectively. $\Delta \mathrm{T}$ 


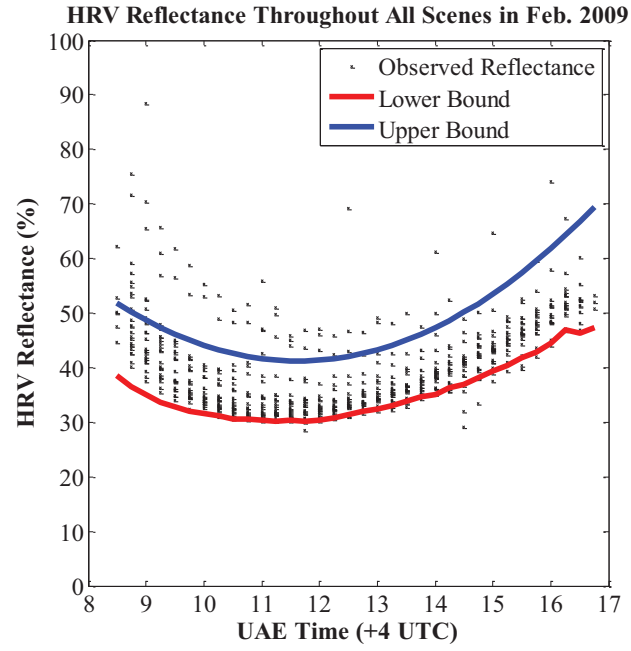

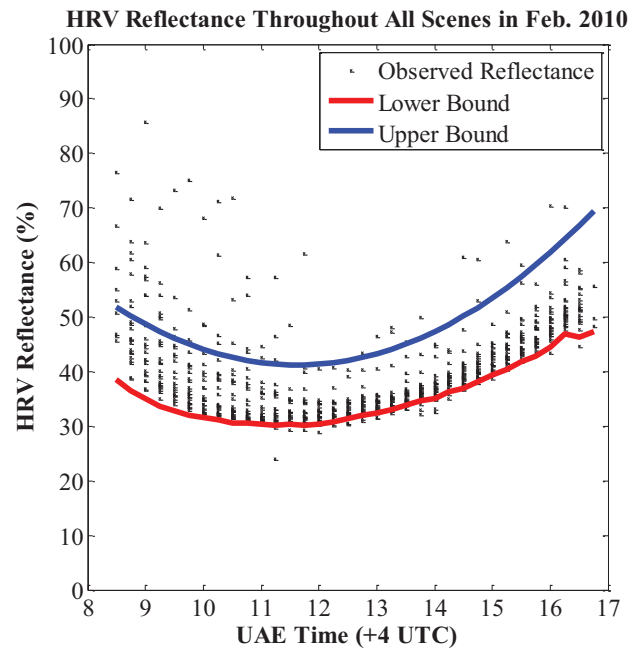

(b)

Fig. 2: The HRV reflectance observations throughout Feb. 2009 (a) and Feb. 2010 (b), upper and lower bounds are shown.

is a pre-defined offset, which is $1.9 \mathrm{~K}$ for barren surfaces [4]. After removing the cloudy pixels by means of the thin cirrus cloud mask, the interception index $i i$ can be derived. It is favorable that the interception index $i i$ has a value between 0 and 1, 0 being for the clearest sky conditions and 1 being for the very dusty conditions in which the $D N I$ is almost $0 \mathrm{~W} / \mathrm{m}^{2}$. Therefore, to derive the interception index $i i$ the following equation will be used:

$$
i i=\frac{\rho^{t}(i, j)-\rho_{\min }^{t}(i, j)}{\rho_{\max -d u s t y}^{t}(i, j)-\rho_{\min }^{t}(i, j)},
$$

where $\rho^{t}(i, j)$ and $\rho_{\text {min }}^{t}(i, j)$ are the same as explained before in Eq. (1) in section 2. $\rho_{\text {max-dusty }}^{t}(i, j)$ is the maximum HRV reflectance occurring in the highest dusty conditions for pixel $(i, j)$, i.e. when the $D N I$ is almost $0 \mathrm{~W} / \mathrm{m}^{2}$. To derive $\rho_{\text {max-dusty }}^{t}(i, j)$, the upper bound of the cloud-free HRV reflectance needs to computed for each scene, location and month. However, care needs to be taken when deriving $\rho_{\text {max-dusty }}^{t}(i, j)$, to make sure that during the chosen month there were dusty days in which the $D N I$ was almost $0 \mathrm{~W} / \mathrm{m}^{2}$.

\section{EXPERIMENTAL SETUP}

The HRV channel observations were available over Masdar City, shown in Fig. 1c, for the month of Feb. for the years 2009 and 2010. Also, available for those same two months are the ground measurements, consisting of the GHI and DNI readings at a $10-\mathrm{min}$ resolution. The ground measurements were carried out using the rotating shadowband pyranometer instrument.

The month of Feb. 2009 is specifically chosen because there were several dusty days occurring during this month, where the $D N I$ was very low. Therefore, it will be simpler to derive $\rho_{\text {max-dusty }}^{t}(i, j)$ for this month due to presence of dusty days. In this study, data from Feb. 2009 will be used to fit $\rho_{\text {max-dusty }}^{t}(i, j)$ with respect to time. It will also be used to correlate the attenuation of the $D N I$ with the interception index ii. That will be done by plotting the DNI clearness index $\left(D N I / I_{0} \varepsilon\right)$ versus the interception index $i i$, where $I_{0}$ is the solar constant having a value of $1,367 \mathrm{~W} / \mathrm{m}^{2}$ and $\varepsilon$ is the eccentricity correction. The eccentricity correction is computed by [8]:

$\varepsilon=1.000110+0.034221 \cos B+0.001280 \sin B$

$+0.000719 \cos 2 B+0.000077 \sin 2 B$,

$B=(j-1) \frac{360}{365}$,

where $B$ is in degrees and $j$ is the day number.

For the data of Feb. 2010 over Masdar City, the interception index $i i$ will be computed for all scenes in that month using the fit of $\rho_{\text {max -dusty }}^{t}(i, j)$, which is derived using the Feb. 2009 data. Also, the DNI for Feb. 2010 will be estimated using the correlation between $D N I / I_{0} \varepsilon$ and the interception index $i i$, derived from Feb. 2009 data. The accuracy of the estimated $D N I$ is based on the relative root mean square error (rRMSE) and the relative mean bias error (rMBE).

\section{RESULTS AND DISCUSSION}

Fig. 2a shows the HRV reflectance observations throughout all the cloud-free scenes for Masdar City station for the month of Feb. 2009. The figure also shows the lower bound, i.e. the surface albedo throughout the month, and the upper bound, i.e. $\rho_{\text {max-dusty }}^{t}(i, j)$. The fit for $\rho_{\max -d u s t y}^{t}(i, j)$ is a $2^{\text {nd }}$ degree polynomial fit.

Fig. $2 \mathrm{~b}$ shows the upper and lower bounds for the HRV reflectance observations throughout the testing month of Feb. 2010 for Masdar City station. The interception index ii is then derived for each scene in the month of Feb. 2010.

There will be interception index $i i$ values less than 0 and greater than 1 , due to the outliers, which are not within the upper and lower bounds shown in Fig. $2 \mathrm{a}$ and $2 \mathrm{~b}$. 


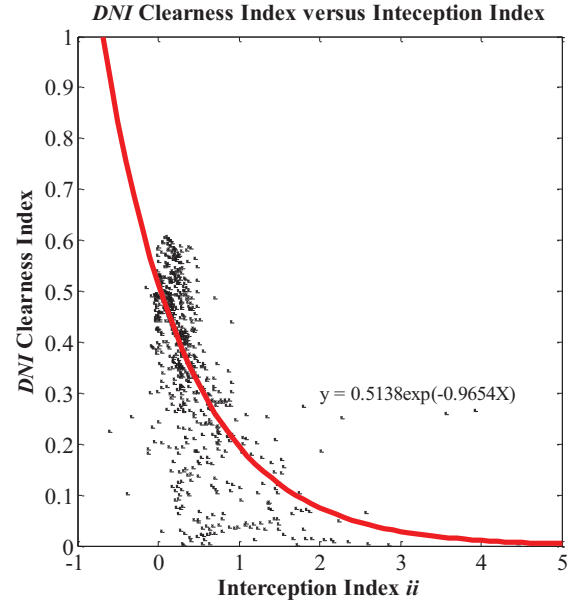

Fig. 3: DNI clearness index versus interception index.

Fig. 3 shows the scatter plot between $D N I / I_{0} \varepsilon$ and the interception index $i i$ for the month of Feb. 2009. The fit with the highest $\mathrm{R}^{2}$ value is simply an exponential fit.

Applying the exponential equation into the data of Feb. 2010 and then computing the DNI at a 15-min temporal resolution, an rRMSE of $32.1 \%$ and an $\mathrm{rMBE}$ of $-2.9 \%$ are obtained. The scatter plot between the estimated $D N I$ and the ground measured DNI for the testing month of Feb. 2010 is shown in Fig. 4.

It is clear that the fit between the estimated and measured $D N I$ has some trends. The higher $D N I$ values are underestimated, while other DNI values are generally overestimated. Even though the HRV channel is sensitive to dust as shown earlier, it is not sensitive to water vapor. Therefore, in area where water vapor is highly variable, like the UAE, the interception index $i i$ will face some difficulties in estimating the DNI. Still, the interception index $i i$ can give an indication of the dust conditions present in the atmosphere.

\section{CONCLUSIONS}

Due to the sensitivity of the HRV SEVIRI channel to dust over bright desert surfaces the interception index $i i$ is proposed. The interception index $i i$ is derived from the HRV channel of SEVIRI and acts as an indicator of the atmospheric dusty conditions. The interception index $i i$ could further be used to derive the DNI. Using testing data from Feb. 2010 over Masdar City in the UAE, the obtained rRMSE and the rMBE for the estimated DNI at a 15 -min temporal resolution are $32.1 \%$ and $-2.9 \%$, respectively.

Future work on this topic include a method to derive the $\rho_{\text {max-dusty }}^{t}(i, j)$ for months which do not encounter heavy dust storms. Also, the correlation between the interception index $i i$ and the DNI attenuation could see some improvements in future work.

However, water vapor will remain as a setback when applying the interception index $i i$ to estimate the $D N I$. Water

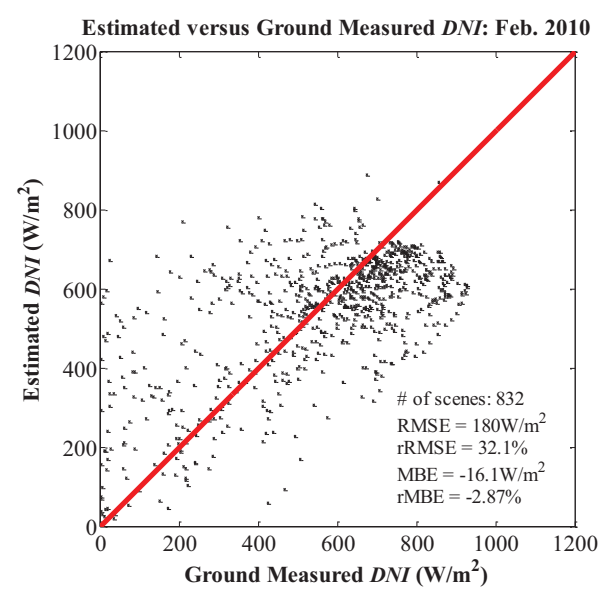

Fig. 4: Estimated versus measured $D N I$ for testing month.

vapor is not detected by the visible satellite channels, but it has a significant effect in solar radiation attenuation.

\section{REFERENCES}

[1] D. Cano, et al., "A Method for the Determination of the Global Solar Radiation from Meteorological Satellite Data," Solar Energy, vol. 37, pp. 31-39, 1986.

[2] A. Hammer, et al., "Solar Energy Assessment using Remote Sensing Technologies," Remote Sensing of Environment, vol. 86, pp. 423-432, 2003.

[3] C. Rigollier, M. Lefèvre and L. Wald, "The Method Heliosat-2 for Deriving Shortwave Solar Radiation from Satellite Images," Solar Energy, vol. 77, pp. 159-169, 2004.

[4] J. Hocking, P. N. Francis and R. Saunders, "Cloud Detection in Meteosat Second Generation Imagery at the Met Office,"

Meteorological Applications, 2010.

[5] E. Dountio, et al., "On the Reliability of HELIOSAT Method: A Comparison with Experimental Data," Solar Energy, vol. 84, pp. 1047-1058, 2010.

[6] I. Moradi, et al., "Evaluation of the Heliosat-II Method using Daily Irradiation Data for Four Stations in Iran," Solar Energy, vol. 83, pp. 150-156, 2009.

[7] R. Perez, et al., "A New Operational Model for SatelliteDerived Irradiances: Description and Validation," Solar Energy, vol. 73, pp. 307-317, 2002.

[8] J.A. Duffie and W.A. Beckman, Solar Engineering of Thermal Processes, John Wiley \& Sons, Inc., USA, 2006. 